\section{Easy Demonstration of Nuclear Division in an Animal Cell}

THE chance appearance of a perfectly stained nematode egg in a smear of intestinal Protozoa prepared by a student (Marjorie Ind) led to the making of preparations $a d$ hoc. The details of nuclear division in these were clear, and as cytology is strange ground to me I consulted Dr. C. D. Darlington.

The worm first treated was Nematospiroides dubius Bay. (Strongyloidea), kindly identified by Dr. H. A. Baylis. It is a very common parasite of the upper part of the small intestine of the woodmouse, Apodemus sylvaticus, pink in colour, coiled into a characteristic tight spiral column, the female worm being about $10 \mathrm{~mm}$. in length. The eggs segment to eight cells in utero. A mouse may yield more than a hundred worms, and in the colony in my garden all seem to be infected. They are caught by a box trap with oatmeal on the floor and a raisin on the trigger.

Owing to the large size of the eggs of the worm the treatment is rather slow. Put one to four female worms on a coverslip with just enough saline to hold them; cut across repeatedly with needles to release eggs and pieces of oviduct containing eggs and sperm; smear with a stroke of the needle; float film downwards on to freshly mixed alcoholic Bouin's fluid; reverse after a minute and fix for three hours. The smear is allowed thirty minutes at each alcohol change and staining is by Delafield's hæmatoxylin, port wine colour, overnight. Differentiate with 1 per cent acid alcohol for three hours; blue the film in slightly ammoniated alcohol of the same grade; clear with clove oil for thirty minutes and allow a few minutes in xylol before mounting.

The common strongyloid worm, Oswaldocruzia sp., of the frog intestine answers equally well, and no doubt many other small nematode worms will do so. This opens up a field of investigation, as Dr. Darlington says that, in spite of the classical example of Ascaris, very little is known about the chromosomes of nematodes. The clearest picture is given of meiosis in the maturing eggs, a series of several often lying in a piece of oviduct and showing successive steps. There are six bivalents all showing terminal chiasmata, and at late metaphase these appear like a circle of large diplococci which can be counted with the $1 / 6$ objective (see photomicrograph). Mitosis also can be followed in the segmenting egg, but is less clear in detail than meiosis as the chromosomes appear

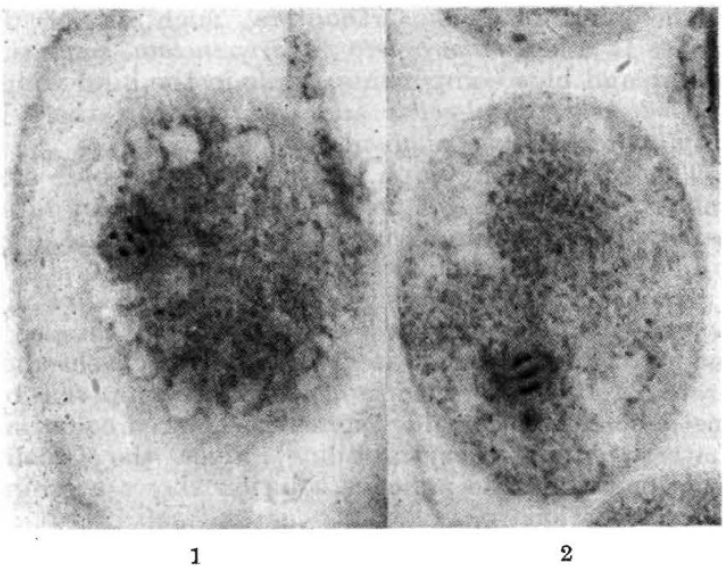

MatuRING gGas of Nematospiroides dubius (NEMATODA) SHOWING THE NUOLEUS IN MTEIOSIB; POLAR AND SIDE VIEWS. $\times 500$. PHOTO. BY J. MANBY. as a ring, or double ring, of minute granules on a quite clear spindle. Chromosome behaviour is much the same in both the species of worms examined. Dr. Darlington considers that a study of meiosis will prove more important than the mitosis which does not show the features (that is, diminution) of the Ascaris type.

Methods of preparing smears for chromosomes are, of course, well known, but the application to nematode worms appears to be new, or not generally known. It is for its possibilities for class work that this casual discovery makes its appeal to me. The zoologist need no longer go to plants for the demonstration of nuclear division. The worms from wild mice, voles, frogs or toads are available at any time of the year. No skilled dissection is required and the technique is simple. The student who has helped me with the preparations was successful in her first attempts with little conning. It is hoped that some worker who has access to living hookworms, Ancylostoma and Necator, will make tests with these and report on the results. The eggs are very similar to those of the worms here dealt with and should be even more readily manipulated.

The University,

$$
\text { LL. LLOYD. }
$$

Leeds, 2. March 17.

\section{Species of Lucilia Attacking Sheep in South Africa}

Two species of Lucilia are stated to be associated in the sheep blowfly complex in South Africa. Of these, Lucilia cuprina Wied. is regarded as the main primary fly which produces strike in living shoop. $L$. sericata Mg., on the other hand, is not considered as important in this respect, although known to be the chief cause of strikes in Europe.

The systematic separation of the two species rests mainly upon a difference in the colour of the femur of the fore-leg in the adult fly. In $L$. cuprina this is bright green ; in L. sericata it is black. In L. cuprina the abdomen is green with a bronze sheen; in a typical $L$. sericata fly it is a distinct blue-green.

In any batch of field material containing both species, there is nearly always a gradation in the colour of both femur and abdomen so that, with certain individuals, a correct classification is difficult. The colour of a fly can also vary with the composition of the larval food supply. In addition, colour is often misleading, and in some cases is largely dependent upon the opinion of the observer. It is therefore not a scientific criterion.

The two species of flies are biologically identical. Both are attracted to, and breed upon, carrion, and both occur in wounds on the living sheep. Moreover, they interbreed readily in captivity and there is little doubt that they also do so in Nature.

In breeding experiments carried out at this Lab. oratory, crosses were made in both directions which gave rise to fertile progeny. In the $F_{1}$ generation, the green femur of L. cuprina and the blue-green abdomen of $L$. sericata proved to be dominant, all the progeny having these two characters. In the $F_{2}$ generation, segregation occurred, giving individuals of three types, namely, (a) with green femur and bronzegreen abdomen (typical L. cuprina); (b) with green femur and blue-green abdomen (hybrids); (c) with black femur and blue-green abdomen (typical $L$. sericata).

In a typical batch of $F_{2}$ progeny from such a 